\title{
X-ray properties of the transient pulsar 3A $0535+262$ in quiescence
}

\author{
U. Mukherjee and B. Paul
}

\author{
Department of Astronomy and Astrophysics, Tata Institute of Fundamental Research, Homi Bhabha Road, 400005 Colaba, \\ Mumbai, India \\ e-mail: [uddipan;bpaul]@tifr.res.in
}

Received 15 July 2004 / Accepted 27 October 2004

\begin{abstract}
We present the timing and spectral properties of the transient Be/X-ray binary pulsar 3A 0535+262 during quiescence using three observations with the narrow field imaging instruments (NFI) of BeppoSAX. Assuming a distance of $2 \mathrm{kpc}$ for this system, the 2-10 keV X-ray luminosities measured from the three observations are in the range of $1.5-4.0 \times 10^{33} \mathrm{erg} \mathrm{s}^{-1}$, indicating a very low rate of accretion. We report the detection of pulsations at a very low luminosity of $2 \times 10^{33} \mathrm{erg} \mathrm{s}^{-1} \mathrm{during}$ one of the three observations, though at this accretion rate the system is expected to be in the centrifugally inhibited regime. The X-ray spectra for the unpulsed observations are best modeled as power law type while a combined model of power law and black-body is required to fit the pulsed spectrum.
\end{abstract}

Key words. stars: pulsars: individual: 3A 0535+262 - stars: neutron - stars: circumstellar matter - X-rays: general X-rays: stars

\section{Introduction}

3A $0535+262$ is a close binary system which contains a 09.7 IIIe Be star (HDE 245770, Giangrande et al. 1980) and a slowly rotating, strongly magnetized neutron star. The X-ray source was discovered by the Ariel V satellite (Rosenberg et al. 1975) when it was in a "giant outburst" mode with X-ray intensity of the order of $\sim 2$ Crab in the 3-7 keV range and showed pulsations at $104 \mathrm{~s}$. The system has been observed to undergo several transient X-ray outbursts classified as "giant" $(2-10 \mathrm{keV}$ flux $>1 \mathrm{crab})$ and "normal" $(2-10 \mathrm{keV}$ flux $<1 \mathrm{crab})$ outbursts. After 1975, two more giant outbursts were observed, one in 1980 detected with the Hakucho satellite (Nagase et al. 1982) and another one in 1994 by the BATSE detectors onboard CGRO (Finger et al. 1996). A large orbital period of $111.0 \pm 0.4$ days was discovered from the long term light curves obtained with the Vela 5B satellite (Priedhorsky and Terrell 1983). Interestingly, the system shows significant periodic optical variability at a different period of $\sim 103$ days (Larionov et al. 2001). During the 1994 outburst, quasi-periodic oscillations (QPO) were observed with frequency correlated to the X-ray flux and spin-up torque (Finger et al. 1996, Ghosh 1996). The orbital parameters were also determined from observations made with BATSE during this outburst. The magnetic field strength of the neutron star in 3A $0535+262$ is $\sim 1 \times 10^{13}$ Gauss as measured from the cyclotron resonance scattering feature with the OSSE experiment onboard CGRO during the 1994 outburst (Grove et al. 1995).
With EXOSAT observations made in 1985-1986 in between the outbursts, a moderate and pulsating X-ray flux was detected from 3A 0535+262 at various orbital phases (Motch et al. 1991). The X-ray flux in the $1-20 \mathrm{keV}$ energy band was measured to be in the range of $1.5-3.0 \times 10^{-10} \mathrm{erg} \mathrm{cm}^{-2} \mathrm{~s}^{-1}$, which corresponds to a luminosity of $0.7-1.4 \times 10^{35} \mathrm{erg} \mathrm{s}^{-1}$ for a distance of $2 \mathrm{kpc}$ (Steele et al. 1998). The detection of $\mathrm{X}$-ray pulsations in two of the EXOSAT observations showed that the mass accretion rate was large enough not to cause centrifugal inhibition and that material from the outflow of the Be star companion accretes through a disk onto the neutron star. In more recent observations of 3A $0535+262$ made with the Proportional Counter Array of the Rossi X-ray Timing Explorer (RXTE-PCA), the source was detected at a much lower luminosity of $2.0-4.5 \times 10^{33} \mathrm{erg} \mathrm{s}^{-1}$ at which the onset of centrifugal inhibition of accretion or propeller effect is expected. The $\mathrm{X}$-ray luminosity that was still detected in the propeller regime was ascribed to material leaking through the magnetosphere or thermal emission from the heated core of the neutron star (Negueruela et al. 2000). However, as the RXTE-PCA detectors are non-imaging instruments and therefore have large internal and X-ray sky background, the flux and spectral measurements at low intensity are not very accurate.

In this paper, we present $\mathrm{X}$-ray timing and spectral properties of $3 \mathrm{~A} 0535+262$ in a quiescent state from three observations made with the Beppo-SAX narrow field imaging spectrometer instruments. Detection of pulsations and measurement of the X-ray spectrum in quiescent state may resolve 
the outstanding issue regarding the different accretion regimes in which the system would reside. In the subsequent sections we describe the observations (Sect. 2), results from timing and spectral analysis (Sect. 3) and a discussion on the propeller effect in this pulsar and the X-ray spectral properties in the pulsating and non-pulsating states (Sect. 4) based on the results obtained here.

\section{Observations}

Three observations of $3 \mathrm{~A} 0535+262$ were made with the Beppo-SAX observatory on 2000 September 4 (5:14 UT), October 5 (00:42 UT) and 2001 March 5 (22:52 UT) (observations $\mathrm{A}, \mathrm{B}$ and $\mathrm{C}$ hereafter). The scientific payload of BeppoSAX consists of four Narrow Field Instruments (NFI) comprising one unit of Low Energy Concentrator Spectrometer (LECS; $\left.0.1-10 \mathrm{keV}, 22 \mathrm{~cm}^{2} @ 0.28 \mathrm{keV}\right)$ and three Medium Energy Concentrator Spectrometer (MECS; $1.3-10 \mathrm{keV}, 150 \mathrm{~cm}^{2} @$ $6 \mathrm{keV})$. The energy resolution (\% FWHM) of the LECS and the MECS detectors is $8 \times(E / 6 \mathrm{keV})^{-0.5}$. BeppoSAX also consists of a High Pressure Gas Scintillator Proportional Counter alongwith a Phoswich Detection System. A detailed description of Beppo-SAX can be found in Boella et al. (1997). Taking the time of periastron passage and the orbital period from Finger et al. (1996), the orbital phases of the three observations A, $\mathrm{B}$ and $\mathrm{C}$ are $0.77,0.05$ and 0.42 respectively. The respective observations are shown in Fig. 1 along-with a sketch of the orbit of 3A $0535+262 / \mathrm{HDE} 245770$ binary system. The useful on-source times for the three observations were $32 \mathrm{ks}, 39 \mathrm{ks}$ and $51 \mathrm{ks}$ each for the MECS and $19 \mathrm{ks}, 30 \mathrm{ks}$ and $43 \mathrm{ks}$ for the LECS detectors. As the source 3A $0535+262$ was very faint during these observations, we have analyzed data only from the narrow field imaging instruments. Data from only two of the MECS detectors were available and the event files of the two detectors were added before further analysis.

\section{Data analysis}

\subsection{Timing analysis}

We extracted the MECS light curves with a time resolution of $1.0 \mathrm{~s}$ and then corrected the arrival times to the times at the solar system barycentre. The average count rate of the light curves of $\mathrm{A}$ and $\mathrm{B}$ were $\sim 0.04$ count $\mathrm{s}^{-1}$ while that of $\mathrm{C}$ was 0.09 count $\mathrm{s}^{-1}$. All the three light curves showed intensity variations by more than a factor of two at a few hours to day timescale. The light curve of $\mathrm{C}$ had a stretch of $240 \mathrm{ks}$ spread over several satellite orbits, which is useful for accurate measurement of pulse period. We searched for pulsations in the light curves of all the three observations using the pulse folding and $\chi^{2}$ maximization technique in the expected pulse period range of $102-104 \mathrm{~s}$ with a period resolution of $0.0035 \mathrm{~s}$. Only the observation $\mathrm{C}$ showed coherent pulsations with a period of 103.41 s and the result of the period search is shown in Fig. 2. Another period of $101.57 \mathrm{~s}$ also showed up when the period search was performed over a wider range between 101-105 s. But by looking at the long term pulse period history of this pulsar (Fig. 3), it may be inferred that a period of $101.57 \mathrm{~s}$ is

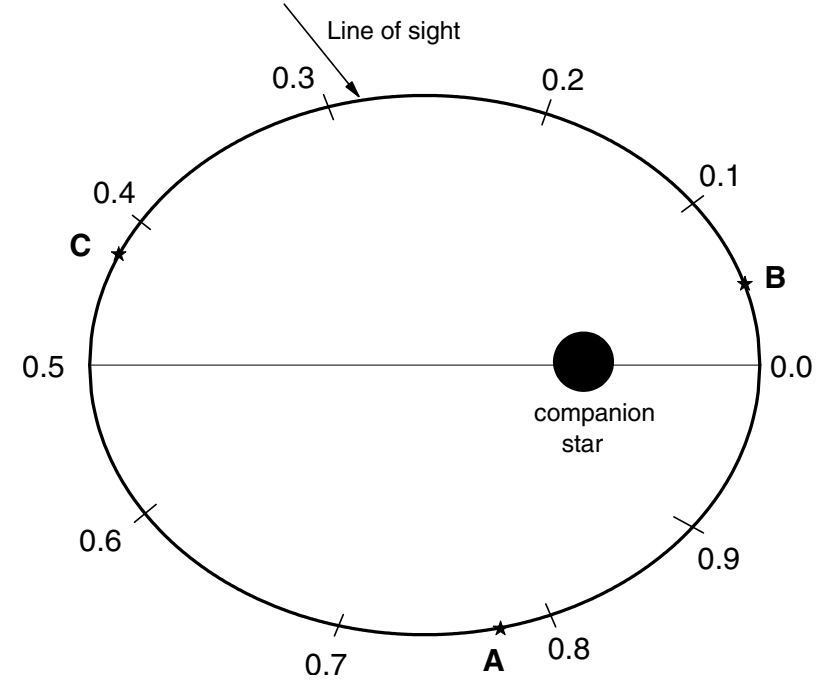

Fig. 1. Orbital geometry of 3A $0535+262 /$ HDE 245770 binary system. The orbital phases are marked on the circumference of the ellipse. The observations A, B and C are denoted with asterisks and the projection of the line of sight on the orbital plane is also shown. The orbital parameters are taken from Finger et al. (1996). The radius of the companion star is $15 R_{\odot}$ and the inclination of the orbit is $\sim 25^{\circ}$ (Okazaki \& Negueruela 2001)

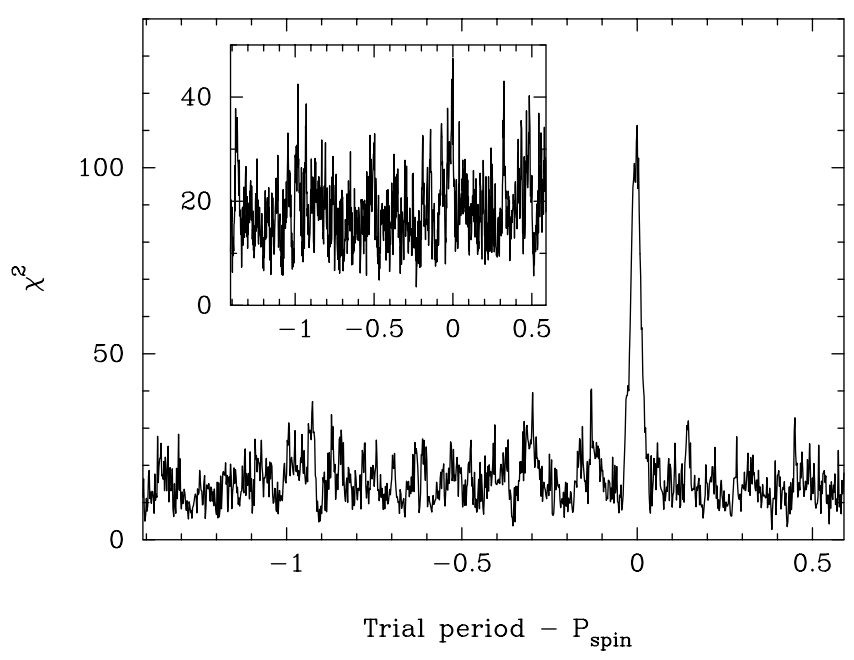

Fig. 2. The results of the period search for the third observation with the inset showing the period search result for the portion of the lightcurve with average count rate less than 0.08 count s ${ }^{-1}$.

quite unlikely and arose due to the window function of the light curve.

To determine the lowest intensity in observation $\mathrm{C}$ at which pulsations can be detected from $3 \mathrm{~A} 0535+26$, we subsequently divided the total MECS observation duration into twenty four roughly equal segments. Following this we extracted five separate light-curves each containing data only from those segments with average count rate less than 0.15 count $\mathrm{s}^{-1}, 0.125$ count $\mathrm{s}^{-1}, 0.1$ count $\mathrm{s}^{-1}, 0.08$ count $\mathrm{s}^{-1}$ and 0.06 count $\mathrm{s}^{-1}$ respectively. From independent pulsation searches on these light curves with the same parameter space as mentioned above, we detected pulsations down to an average count rate of less than 0.08 count $\mathrm{s}^{-1}$. The inset in 


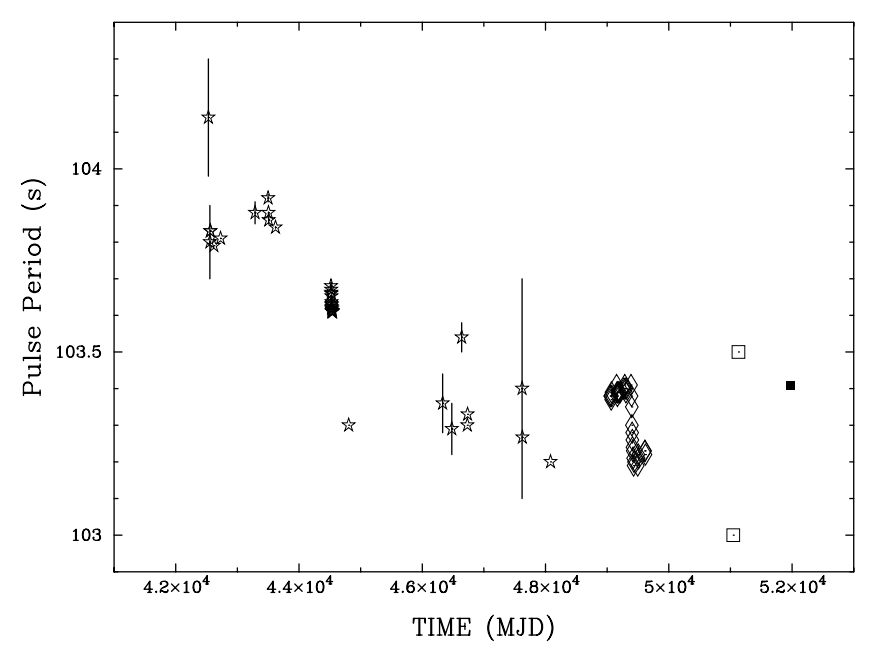

Fig. 3. The long term pulse period history of 3A $0535+262$ taken from Coe et al. (1990) and other references cited in the text. The diamonds represent the BATSE observations by Finger et al. (1996), the open squares show the two RXTE-PCA observations by Negueruela et al. (2000) and the filled square denotes the observation C with BeppoSAX (MJD 51 973).

Fig. 2 shows the period search result of the light-curve created from the segments with average count rate less than 0.08 count $\mathrm{s}^{-1}$. The light curve with segment-wise average count rate of less than 0.06 count $\mathrm{s}^{-1}$ has a very small time duration and pulsations could not be detected independently from it. However, if this light curve is folded with the same pulse period as determined from the entire observation, the pulse profile clearly shows intensity variations correlated with the pulse profiles from the higher intensity segments (Fig. 4). The pulse profiles are background subtracted and the pulse fraction is [(Maximum - Minimum)/Maximum] 50\%. Thus it is fair to conclude that pulsations exist in $3 \mathrm{~A} 0535+262$ during observation $\mathrm{C}$ down to at least a count rate of 0.06 count $\mathrm{s}^{-1}$. Measurement of the X-ray luminosity of 3A $0535+262$ corresponding to this count rate is presented in the next section.

\subsection{Spectral analysis}

The source and background spectra were extracted from the MECS and LECS detectors using circular regions of radius $4^{\prime}$ and $8^{\prime}$ respectively. The energy range chosen for MECS was $1.8-10.5 \mathrm{keV}$ while that for LECS was $0.3-4.5 \mathrm{keV}$, in which the respective instruments have large effective area and the spectral responses are well understood. For all the three observations, the MECS and LECS phase averaged spectra were fitted simultaneously, with the relative normalization of the two instruments allowed to vary. All the spectra were suitably binned to allow use of $\chi^{2}$ statistics.

The continuum spectra of accreting X-ray pulsars are most often described by a power law with a high energy cut-off, with the cut-off energy usually above $10 \mathrm{keV}$. Therefore, while fitting a pulsar spectra in an energy band that is not very wide, only a power law or a black-body model is invoked. Orlandini $\&$ Dal Fiume (2001) noted that the continuum of accreting $\mathrm{X}$-ray pulsars as observed by BeppoSAX can be well described

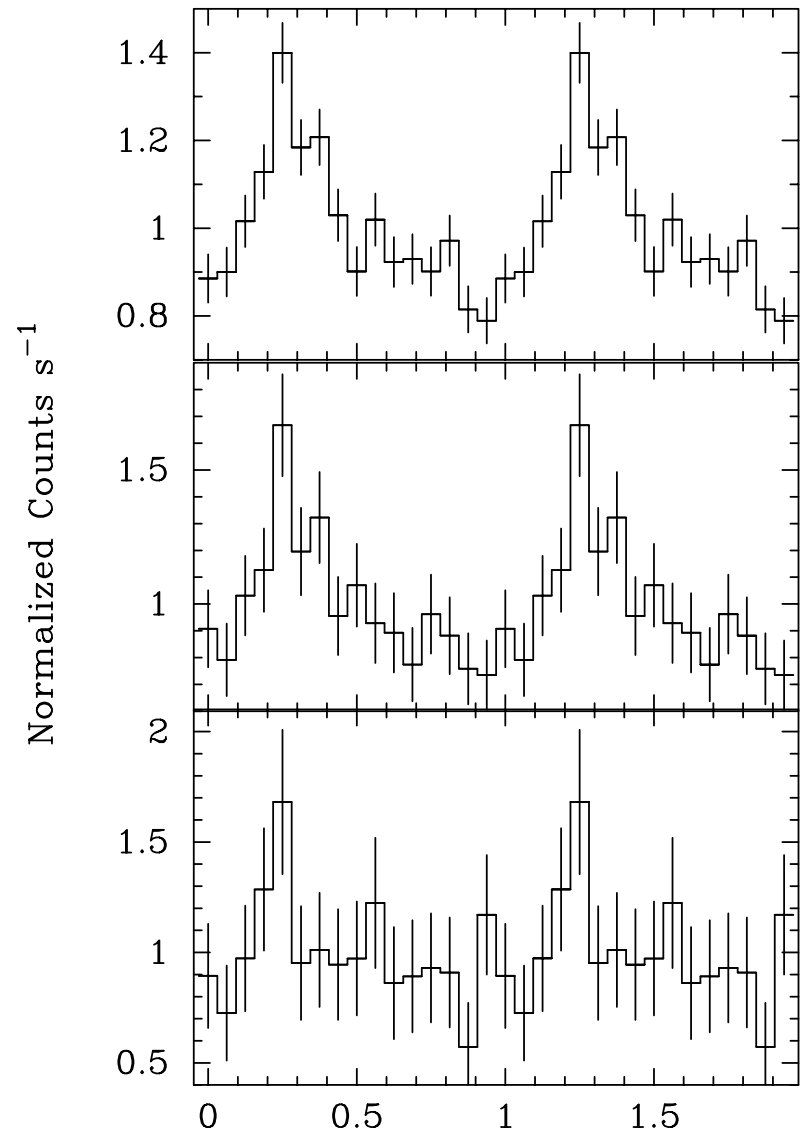

Phase

Fig. 4. The top panel shows the pulse profile for the entire duration of observation $\mathrm{C}$. The middle panel shows the pulse profile for the part of the lightcurve of observation $\mathrm{C}$ in which the count rate is less than 0.08 count $\mathrm{s}^{-1}$ and the bottom panel shows the pulse profile of the part of the lightcurve of $\mathrm{C}$ in which the count rate is less than 0.06 count $\mathrm{s}^{-1}$.

in terms of a black-body component, a power law and a high energy cutoff $\left(E_{\mathrm{c}}>10 \mathrm{keV}\right)$. The main physical process responsible for the continuum emission in accreting X-ray pulsars is Compton up-scattering of soft seed photons. Depending on the value of the comptonization parameter $(y)$, the emergent spectrum of the photons can be a black-body type $(y \ll 1)$ or power law type with a high energy cut-off (if $y \gg 1$ and the inverse compton scattering is unsaturated). Motivated by these factors, we fitted the spectra in XSPEC v11.2.0 (Shafer et al. 1989) with power law and black-body models along-with an absorption by material in the line of sight. The black-body model does not fit any one of the three spectra well and reduced $\chi^{2}$ of more than 2.0 were obtained. The results from the power law spectral fit are given in Table 1 along with $90 \%$ confidence limits for the spectral parameters. We found that the spectra from the first two observations (A and B) fit very well with the model while the spectrum from observation $C$ during which the $\mathrm{X}$-ray luminosity was higher, does not fit. Hence we tried to put in another additive component. A power law and a black-body component along with absorption column density was found to fit the spectrum of observation $\mathrm{C}$ well and the corresponding 
Table 1. Power law spectral parameters. The luminosities are in the $2-10 \mathrm{keV}$ range for a distance of $2 \mathrm{kpc}$

\begin{tabular}{ccccc}
\hline \hline Obs. & $N_{\mathrm{H}}\left(10^{22} \mathrm{~cm}^{-2}\right)$ & $\operatorname{Index}(\Gamma)$ & $L\left(10^{33} \mathrm{erg} \mathrm{s}^{-1}\right)$ & Red. $\chi^{2}$ (d.o.f.) \\
\hline A & $0.65_{-0.12}^{+0.25}$ & $1.93_{-0.16}^{+0.17}$ & 1.54 & $0.93(44)$ \\
& & & & \\
B & $0.64_{-0.23}^{+0.18}$ & $1.81_{-0.13}^{+0.13}$ & 1.62 & $1.07(44)$ \\
& & & & \\
C & $0.69_{-0.12}^{+0.12}$ & $1.69_{-0.08}^{+0.07}$ & 4.07 & $1.71(44)$ \\
\hline
\end{tabular}

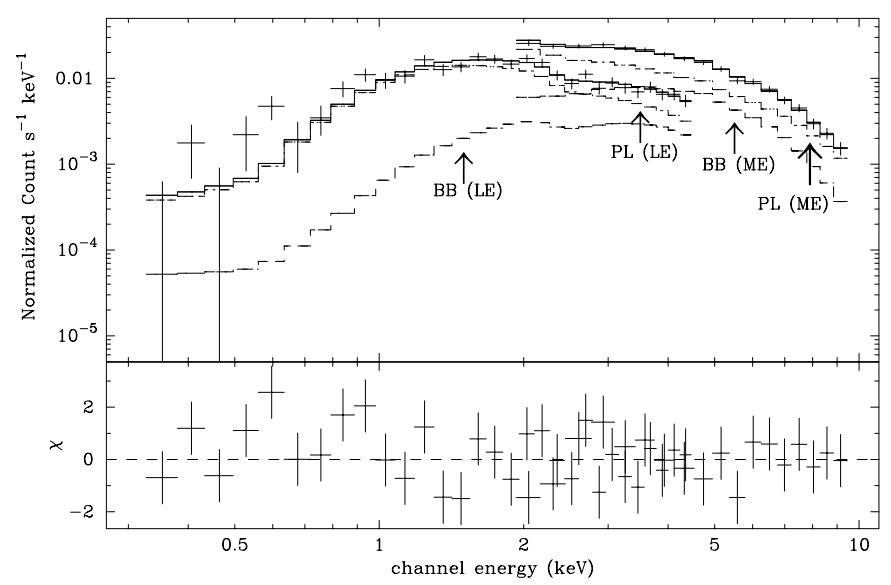

Fig. 5. The fitted spectrum of observation $\mathrm{C}$ along with the residuals in the lower panel. The individual components of the spectral model are also shown (ME: MECS, LE: LECS, PL: Power Law and BB: Black-Body)

spectral parameters are given in Table 2. The power law photon index (for a single power law component for observations A and $\mathrm{B}$ and a double component model for observation $\mathrm{C}$ ) during these three observations is between 1.5-2.0, whereas the temperature of the black-body component is $1.33 \mathrm{keV}$. Assuming a $2 \mathrm{kpc}$ distance for the object, the radius of the emission region for this black-body model is estimated to be $\sim 0.1 \mathrm{~km}$. The absorption column density, for all the three observations is about $6.5 \times 10^{21}$ atoms $\mathrm{cm}^{-2}$, marginally above the galactic column density towards $3 \mathrm{~A} 0535+262$, which is $5.9 \times 10^{21}$ atoms $\mathrm{cm}^{-2}$. The 2-10 keV luminosities (in unit of $10^{33} \mathrm{erg} \mathrm{s}^{-1}$ ) for observations $\mathrm{A}, \mathrm{B}$ and $\mathrm{C}$ are $1.54,1.62$ and 4.07 respectively for power law type spectra and 3.93 for the two component spectrum in observation $\mathrm{C}$. The spectrum from observation $\mathrm{C}$ is shown in Fig. 5, along with the model components and the residual to the best fit model.

The LECS and MECS spectra were extracted for the time intervals in observation $\mathrm{C}$ during which the average count rate was less than 0.06 count $\mathrm{s}^{-1}$ (see the previous section). These spectra were fitted simultaneously with the power law plus black-body model with the absorption column density, power law photon index, black-body temperature, and the relative LECS/MECS normalization being kept fixed to the values determined from the entire observation. Thus the lowest luminosity at which pulsations could be detected from 3A $0535+262$ came out to be $2.0 \times 10^{33} \mathrm{erg} \mathrm{s}^{-1}$ calculated for a distance of $2 \mathrm{kpc}$.

\section{Discussion}

Transient X-ray pulsars in general are prone to wide variations in mass accretion rates and are suitable systems to test the various accretion regimes onto high magnetic field neutron stars. They generally have luminosities of the order of $10^{32}-10^{33} \mathrm{erg} \mathrm{s}^{-1}$ during quiescence. Detection of pulsations in these sources with such a low luminosity seems to be an interesting phenomenon regarding the classification of the accretion regimes in which these systems may exist.

The luminosities obtained by us, $1.5-4.0 \times 10^{33} \mathrm{erg} \mathrm{s}^{-1}$, during the three quiescence observations with BeppoSAX are similar to that of Negueruela et al. (2000) measured with the RXTE-PCA in quiescence. But the EXOSAT observations of this source during previous low states (Motch et al. 1991) report a luminosity two orders of magnitude higher than what is observed in quiescence. During observation $C$, we show that pulsations exist for a luminosity as low as $2.0 \times 10^{33} \mathrm{erg} \mathrm{s}^{-1}$, similar to the lowest luminosity obtained for this source in quiescence by Negueruela et al. (2000). However, as the RXTEPCA detectors are non-imaging instruments, the diffuse X-ray emission from the galactic ridge can cause hindrance to an accurate measurement of the very low X-ray flux and the spectral properties of $3 \mathrm{~A} 0535+262$ in quiescence.

Assuming an efficiency $\eta=1$ in the conversion of gravitational energy into X-ray luminosity, the luminosity obtained by us in the whole of observation $\mathrm{C}\left(\ell=3.95 \times 10^{33} \mathrm{erg} \mathrm{s}^{-1}\right)$ gives an accretion rate $\dot{M}=2.05 \times 10^{13} \mathrm{~g} \mathrm{~s}^{-1}$. For the known surface magnetic field strength and the spin period of this pulsar, we obtain the magnetospheric radius as

$r_{\mathrm{m}}=9.35 \times 10^{9} \mathrm{~cm}$

and the corotation radius is calculated to be

$r_{\mathrm{c}}=3.0 \times 10^{9} \mathrm{~cm}$.

Therefore as $r_{\mathrm{m}}>r_{\mathrm{c}}$, following Stella et al. (1986), the pulsar in observation $\mathrm{C}$ as well as in A and B can be expected to be in the centrifugally inhibited regime. In this regime, accretion onto the neutron star surface is inhibited by the centrifugal action of the rotating magnetosphere (Illarionov \& Sunyaev 1975). As observations A and B do not show pulsations, we infer that at such a low level of luminosity they are indeed in the centrifugally inhibited regime. During observation $\mathrm{C}$, which has the highest luminosity among the three, though $r_{\mathrm{m}}>r_{\mathrm{c}}$ would mean the existence of a centrifugal barrier, pulsations have been detected unambiguously. The detection of pulsations in $\mathrm{C}$ probably means that a fraction of the disk material is somehow going onto the surface of the neutron star along the magnetic field lines. The three BeppoSAX NFI observations combined together show that at a luminosity between 1.5-4.0 $\times$ $10^{33} \mathrm{erg} \mathrm{s}^{-1}$, 3A $0535+262$ makes a transition between centrifugal inhibition and direct accretion onto the neutron star surface. We would like to note here that there are some uncertainties regarding the calculation of $r_{\mathrm{m}}$. If $\eta$ is not equal to 1 
Table 2. Power law + black body spectral parameters for Obs. C. (The quoted luminosities are in the $2-10 \mathrm{keV}$ range and assuming a distance of $2 \mathrm{kpc} ; L_{\mathrm{BB}}$ and $L_{\mathrm{PL}}$ are the luminoisities corresponding to the black body and power law components.)

\begin{tabular}{ccccccc}
\hline \hline$N_{\mathrm{H}}\left(10^{22} \mathrm{~cm}^{-2}\right)$ & $\operatorname{Index}(\Gamma)$ & $k T(\mathrm{keV})$ & $R(\mathrm{~km})$ & $L_{\mathrm{BB}}\left(10^{33} \mathrm{erg} \mathrm{s}^{-1}\right)$ & $L_{P L}\left(10^{33} \mathrm{erg} \mathrm{s}^{-1}\right)$ & Red. $\chi^{2}$ (d.o.f.) \\
\hline $0.6_{-0.6}^{+0.6}$ & $1.84_{-0.18}^{+0.34}$ & $1.33_{-0.2}^{+0.17}$ & 0.08 & 1.36 & 2.57 & $1.05(42)$ \\
\hline
\end{tabular}

or if the distance to the source is very different from what has been assumed; the value of $r_{\mathrm{m}}$ would change. A very low $\eta$ would correspond to higher accretion rate which in turn compresses the magnetosphere to give a smaller value of $r_{\mathrm{m}}$. But even with $\eta \sim 0.1$ the value of $r_{\mathrm{m}}$ does not come out to be less than $r_{\mathrm{c}}$. Similarly, a larger distance would imply a larger X-ray luminosity and higher accretion rate. The distance of $2 \mathrm{kpc}$ to $3 \mathrm{~A} 0535+262$ has been determined using spectral type and reddening of its companion star (Steele et al. 1998) and is unlikely to be wrong by a large factor. Another uncertainty in the calculation of $r_{\mathrm{m}}$ is due to the assumption of aligned rotator. Other X-ray transients like 4U 0115+63 (Campana et al. 2001 with BeppoSAX) and GRO J1744-28 (Wijnands et al. 2002 with Chandra) in quiescence with luminosities of $\sim 10^{33} \mathrm{erg} \mathrm{s}^{-1}$ have also been thought to be in the centrifugally inhibited regime.

We found that the phase averaged spectrum for observation $\mathrm{C}$ fitted well when a combined model of power law and blackbody along with absorption was used. Else in the spectra of the other two observations $\mathrm{A}$ and $\mathrm{B}$, the power law index $(\Gamma)$ was between 1.5-2.0 and the equivalent hydrogen column density obtained was of the order of $10^{21}$ atoms $\mathrm{cm}^{-2}$. The spectral parameters of Motch et al. (1991) are in conformity with ours as they obtained photon index $\sim 1.66$ while the column density was $\sim 10^{21}$ atoms $\mathrm{cm}^{-2}$. The low column density measured for this source matches with the amount of material expected in the line of sight from reddening. The visual extinction $A_{\mathrm{v}} \sim 2.7$ mag (Giangrande et al. 1980) gives a column density of $\sim(4.8-6.0) \times 10^{21}$ atoms $\mathrm{cm}^{-2}$ (Predehl \& Schmitt 1995; Gorenstein 1975). On the other hand, Negueruela et al. (2000) obtained values of the column density which are an order of magnitude higher than that obtained here with the BeppoSAX spectrometers. Since the energy range for RXTE-PCA was taken to be 3-20 keV, the measurements of column density by Negueruela et al. (2000) cannot be compared with the values obtained here with combined BeppoSAX MECS-LECS which goes down to $0.3 \mathrm{keV}$. As the low energy part of the spectrum is measured quite well with BeppoSAX, it can also be used to have some gross understanding about the wind density and the mass-loss rate of the companion star. After taking into account the galactic column density towards this source $(5.9 \times$ $10^{21}$ atoms $\mathrm{cm}^{-2}$ ) it is evident that the column density due to local material is very low; of the order of $10^{20}$ atoms $\mathrm{cm}^{-2}$. A very low column density would imply a very low mass-loss rate for the companion star which can cause the low mass accretion rate as observed here. To have an order of magnitude estimation of the parameters, we have calculated the column density profile with orbital phase for the pulsar by considering a spherically symmetric stellar wind emanating from the companion and assuming a terminal wind velocity of $\sim 1000 \mathrm{~km} \mathrm{~s}^{-1}$

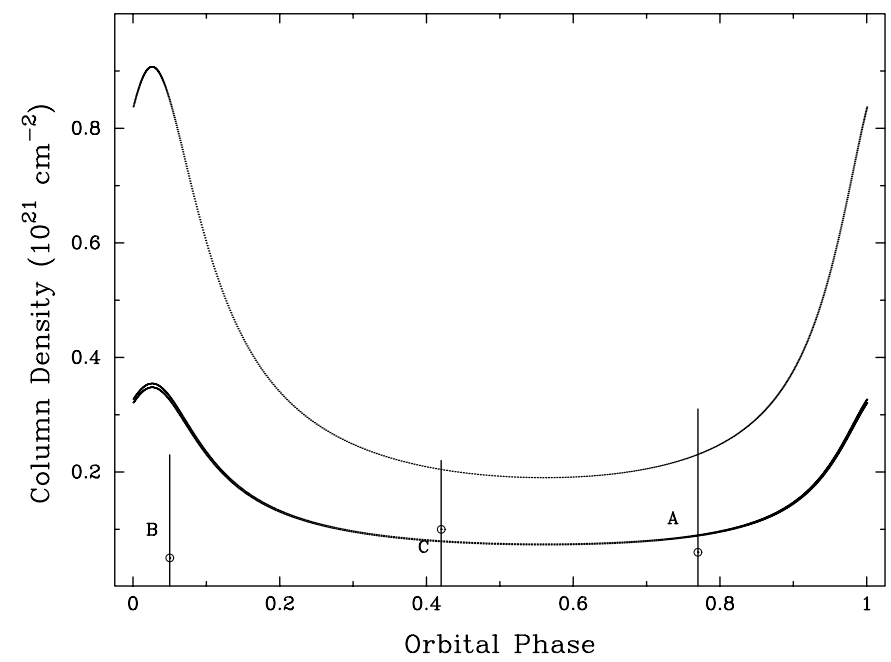

Fig. 6. Model variation of column density with orbital phase calculated for three different mass loss-rates corresponding to the three observations. The observed column densities (see Table 1) after subtracting the galactic column density are superposed along with their error-bars.

and the inclination to the orbit was taken to be $25^{\circ}$ (Okazaki $\&$ Negueruela 2001). Assuming spherical accretion, a set of three curves were obtained, each for the observations A, B \& C with the mass-loss rates being calculated from their respective luminosities. The effective column density values (Observed column density - Galactic column density) as obtained from the power law fits were superimposed on this set of curves as shown in Fig. 6. The effective values of the column density are of the same order of magnitude as of the model. Thus it can be said that a low luminosity and low value of the measured local absorption column density are consistent with a low mass loss rate from the companion star.

It is to be noted that the spectra for the pulsating and the non-pulsating states are different. From the three BeppoSAX observations, it is clear that a power law spectral model fits the quiescent spectra much better than a black-body model, although an additional black-body component helps in improving the fit significantly for observation C. From RXTE-PCA spectrum (Negueruela et al. 2000) it was not possible to distinguish between power law and black-body models. The radii of the emitting region for the black-body component in the third spectrum came out to be almost two orders of magnitude smaller compared to the canonical $10 \mathrm{~km}$ neutron star radius. It is plausible that at very low accretion rates as is the case here, the plasma not having enough ram pressure to compress the magnetosphere moves along the outermost field lines and gets deposited on the very inner parts of the polar caps and hence 
occupy a rather small and compact region. Hence, the blackbody component in the spectra of observation $\mathrm{C}$ with very low emitting region may be possible. In this regard, we mention the RXTE observations of the persistent low luminosity X-ray pulsar 4U 0352+309 (Coburn et al. 2001). The area of the blackbody component $\left(\sim 10^{8} \mathrm{~cm}^{2}\right.$; same as obtained in obs. C) seen in its spectra in low state was consistent with being emitted from the magnetic polar cap of the pulsar.

This black-body component in observation $\mathrm{C}$ is quite different from the soft excess usually observed in some binary X-ray pulsars (Yokogawa et al. 2000, Paul et al. 2002). They are in general modeled as a black-body or thermal bremsstrahlung where the black-body temperature is $\sim 0.1 \mathrm{keV}$ and the area of the emitting region $\sim 10^{15-16} \mathrm{~cm}^{2}$. Recently, Hickox et al. (2004) have explored the physical origin of this so called "soft excess" and found that for sources with luminosity $L_{\mathrm{x}} \leq 10^{36} \mathrm{erg} \mathrm{s}^{-1}$, it may be due to emission by photoionized or collisionally heated diffuse gas, or thermal emission from the surface of the neutron star. In the highly luminous $\left(L_{\mathrm{x}} \geq\right.$ $10^{38} \mathrm{erg} \mathrm{s}^{-1}$ ) sources, the soft component may be due to reprocessing of hard X-rays by the optically thick accreting material, most likely near the inner edge of the accretion disk. At low luminosities, like in the present case, the inner disk temperature can not be high enough for it to be an X-ray emitter. Moreover, the size of the black-body emission region in $3 \mathrm{~A} 0535+262$ is very small compared to that of the inner accretion disk, and the flux in the black-body component is a substantial fraction of that in the power law component ( $\sim 50 \%$ in the present case). These facts suggest that the black-body component is not reprocessed by hard X-ray emission as is the case in many luminous High Mass X-ray Binary (HMXB) pulsars. Rather, this soft black-body component resembles that of $4 \mathrm{U} 0352+309$ (Coburn et al. 2001). In another HMXB, 4U 1700-37, a previously known soft X-ray excess (Haberl et al. 1994) has now been found to consist of a blend of low energy emission lines (Boroson et al. 2003). However, in the cases of 3A 0535+262 (present work) and 4U 0352+309 (Coburn et al. 2001), the temperature of the soft component is an order of magnitude higher and is unlikely to be a blend of narrow emission lines.

\section{Conclusions}

We have investigated the timing and spectral properties of the X-ray pulsar $3 \mathrm{~A} 0535+262$ in quiescent state using the narrow field instruments of the BeppoSAX observatory. We have detected pulsations in observation $\mathrm{C}$ in parts of which the $2-10 \mathrm{keV} \mathrm{X}$-ray luminosity was as low as $2 \times 10^{33} \mathrm{erg} \mathrm{s}^{-1}$. For all the three observations of $3 \mathrm{~A} 0535+262$ the accretion is expected to be centrifugally inhibited though the detection of pulsations at these low flux levels in one of them indicates that some matter may have leaked through onto the neutron star surface. When the emission is non-pulsating, the X-ray spectrum is power law type. The origin of an additional blackbody component in the pulsed observation is not very clear though it seems to be essential in fitting the spectra.

Acknowledgements. We thank the referee L. Kaper for many valuable suggestions, and also the BeppoSAX team for providing the data in the public archive. U.M. thanks K. Sengupta and S. Naik for their help in preparing the manuscript and data analysis. He also acknowledges the Kanwal Rekhi Scholarship of T.I.F.R. Endowment Fund for partial financial support.

\section{References}

Boella, G., Butler, R. C., Perola, G. C., et al. 1997, A\&AS, 122, 299

Boroson, B., Vrtilek, S. D., Kallman, T., \& Corcoran, M. 2003, ApJ, 592,516

Campana, S., Gastaldello, F., Stella, L., et al. 2001, ApJ, 561, 924

Coburn, W., Heindl, W. A., Gruber, D. E., et al. 2001, ApJ, 552, 738

Coe, M. J., Carstairs, I. R., Court, A. J., et al. 1990, MNRAS, 243, 475

Finger, M. H., Wilson R. B., \& Harmon, B. A. 1996, ApJ, 459, 288

Ghosh, P. 1996, ApJ, 459, 244

Giangrande, A., Giovannelli, F., Bartolini, C., Guarnieri, A., \& Piccioni, A. 1980, A\&AS, 40, 289

Gorenstein, P. 1975, ApJ, 198, 95

Grove, J. E., Strickman, M. S., Johnson, W. N., et al. 1995, ApJ, 438, L25

Haberl, F., Aoki, T., \& Mavromatakis, F. 1994, A\&A, 288, 796

Hickox, R. C., Narayan, R., \& Kallman, T. R. 2004 [arXiv: astro-ph/0407115]

Illarionov A. F., \& Sunyaev, R. A. 1975, A\&A, 39, 185

Larionov, V., Lyuty, V. M., \& Zaitseva, G. V. 2001, A\&A, 378, 837

Motch, C., Stella, L., Janot-Pacheco, E., \& Mouchet, M. 1991, ApJ, 369,490

Nagase, F., Hayakawa, S., Kunieda, H. et al. 1982, ApJ, 263, 814

Negueruela, I., Reig, P., Finger, M. H., \& Roche, P. 2000, A\&A, 356, 1003

Okazaki, A. T., \& Negueruela, I. 2001, A\&A, 377, 161

Orlandini, M., \& Dal Fiume, D. 2001, in X-ray astronomy: Stellar Endpoints, AGN, and the Diffuse X-ray Background, ed. White, N. E., Malaguti, G., \& Palumbo, G. G. C., AIP Conf. Proc., 599, 283

Paul, B., Nagase, F., Endo, T., et al. 2002, ApJ, 579, 411

Predehl, P., \& Schmitt, J. H. M. M. 1995, A\&A, 293, 889

Priedhorsky, W. C., \& Terrell. J. 1983, Nature, 303, 681

Rosenberg, F. D., Eyles, C. J., Skinner, G. K., \& Willmore, A. P. 1975, Nature, 226, 628

Shafer, R. A., Haberl, F., \& Arnaud, K. A. 1989, XSPEC: An X-ray Spectral Fitting Package, ESA TM-09 (Paris: ESA)

Steele, I. A., Negueruela, I., Coe, M. J., \& Roche, P. 1998, MNRAS, 297, L5

Stella, L., White, N. E., \& Rosner, R. 1986, ApJ, 308, 669

Wijnands, R., \& Wang, Q. D. 2002, ApJ, 568, L93

Yokogawa, J., Paul, B., Ozaki, M., et al. 2000, ApJ, 539, 191 